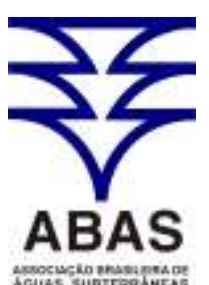

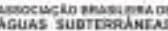
www.abas.org

\section{DESFLUORETAÇÃO DE ÁGUAS SUBTERRÂNEAS UTILIZANDO FILTROS DE CARVÃO ATIVADO DE OSSO}

\author{
DEFLUORIDATION OF GROUNDWATERS USING ACTIVATED BONE \\ CHARCOAL FILTERS
}

\author{
Adilson Ben da Costa ${ }^{1}$, Eduardo Alcayaga Lobo ${ }^{2}$, Jocelene Soares ${ }^{3}$, Alcido Kirst ${ }^{4}$
}

\begin{abstract}
The aim of this study was to develop a domestic filter prototype using activated bone charcoal for the fluoride excess removal in groundwaters for human drinking purposes, evaluating the physical, chemical and microbiological properties of the raw and treated water. The filtration system was constructed using different quantities of activated bone charcoal, confined in a polyvinylchloride tube, working with a discharge of $180 \pm 20 \mathrm{~L} \mathrm{~h}^{-1}$, being treated 10 liters of water per day, enough to supply daily up to five people. The lifetime of this system was established based on Ministry of Health Ordinance $n^{\circ}$ 2914/2011, which limits the fluoride concentration in the water up to $1.5 \mathrm{mg} \mathrm{L}^{-1}$. The results demonstrated the efficiency of the activated bone charcoal in fluoride excess removal, considering a filtration system using $3.5 \mathrm{~kg}$ of this material, enough for meeting the consumption needs for a family of five persons during a period of six months, since observed the assembly and the operation conditions described in this study.
\end{abstract}

Keywords: Fluoride. Activated bone charcoal. Fluorosis. Drink water.

Resumo: O objetivo deste estudo foi desenvolver um protótipo de filtro doméstico usando carvão ativado de osso para a remoção do excesso de íons fluoreto de águas subterrâneas para fins de consumo humano e avaliar as propriedades físicas, químicas, organolépticas e microbiológicas da água bruta e tratada. O sistema de filtração foi construído utilizando diferentes quantidades de carvão ativado de osso, confinado em um tubo de policloroeteno, operando com uma vazão de $180 \pm 20 \mathrm{~L} \mathrm{~h}^{-1}$, e tratando 10 litros de água por dia, o suficiente para o abastecimento diário de uma família de até cinco pessoas. A vida útil deste sistema foi estabelecida com base na Portaria $\mathrm{n}^{\circ} 2914 / 2011$, Ministério da Saúde do Brasil, que limita a concentração de flúor na água até $1,5 \mathrm{mg} \mathrm{L}^{-1}$, indicando a saturação do meio adsorvente. Os resultados demonstraram a eficiência do carvão ativado de osso na remoção do excesso de íons fluoreto, indicando que um sistema de filtração utilizando $3,5 \mathrm{~kg}$ deste material atende às necessidades de consumo de uma família de cinco pessoas durante um período de seis meses, desde que observadas as condições de montagem e operação descritas neste estudo.

Palavras-chave: Fluoreto. Carvão ativado de osso. Fluorose. Água potável.

\title{
INTRODUÇÃO
}

No Brasil, particularmente no interior do Estado do Rio Grande do Sul, o sistema público de abastecimento de água de muitos municípios limita-se apenas à área urbana, e em virtude disto, as comunidades rurais utilizam a água proveniente de poços e fontes naturais, denominados sistemas alternativos de abastecimento, os quais são, muitas vezes, administrados pelas próprias comunidades, na forma de cooperativas.

Entretanto, em muitos casos a água utilizada para abastecimento não apresenta condições de ser consumida sem um tratamento prévio, de-

\footnotetext{
${ }^{1}$ Químico Industrial, Doutor em Química Analítica, Professor do Departamento de Biologia e Farmácia e, do Programa de Mestrado em Sistemas e Processos Industriais da Universidade de Santa Cruz do Sul, RS, Brasil (adilson@unisc.br).

${ }^{2}$ Biólogo, Doutor em Ciências Aquáticas, Professor do Departamento de Biologia e Farmácia e, do Programa de Mestrado em Tecnologia Ambiental da Universidade de Santa Cruz do Sul, Rio Grande do Sul, BR(lobo@unisc.br).

${ }^{3}$ Química Industrial, Mestre em Química Analítica, Técnica do Laboratório de Pesquisa em Tecnologias Ambientais da Universidade de Caxias do Sul, Rio Grande do Sul, BR (s.jocelene@gmail.com).

${ }^{4}$ Químico, Mestre em Desenvolvimento Regional, Professor do Departamento de Química e Física da Universidade de Santa Cruz do Sul, Rio Grande do Sul, BR (alcido@unisc.br).
} 
vido ao fato de algumas características físicas, químicas ou microbiológicas apresentarem valores em desacordo com os padrões recomendados para o consumo humano.

Apesar de menos prioritários em relação aos aspectos microbiológicos de qualidade da água, os aspectos químicos são importantes para assegurar a saúde da população frente a problemas crônicos (WHO, 2008). Diversos autores têm relatado que o consumo prolongado de água com concentração excessiva de íons fluoreto, pode resultar, em crianças, numa patologia denominada fluorose dental, e quando em concentrações extremas, a fluorose esquelética (LOBO e COSTA, 1998; CANGUSSU et al., 2002; CATANI et al., 2007; WHO, 2008; CASTILHO et al., 2010; FRAZÃO et al., 2011).

Segundo a Portaria $n^{\circ} 2914$ de 12 de dezembro de 2011, do Ministério da Saúde (BRASIL, 2011), a concentração máxima permitida para íons fluoreto em águas de abastecimento é de $1,5 \mathrm{mg} \mathrm{L}^{-1}$. Contudo, a Secretaria de Saúde do Rio Grande do Sul através da Portaria $n^{\circ} 10$, de 19 de agosto de 1999, com base em estudos específicos, definiu em $0,8 \mathrm{mg} \mathrm{L}^{-1}$ a concentração ideal para íons fluoreto em águas de consumo humano no estado do Rio Grande do Sul, devido à sua ação na inibição da cárie dental, e teores de 0,6 e 0,9 $\mathrm{mg} \mathrm{L}^{-1}$ como limites mínimos e máximos, respectivamente (RIO GRANDE DO SUL, 1999).

A contaminação química da água, particularmente por íons fluoreto, não é de fácil tratamento. Sistemas de desfluoretação de águas subterrâneas têm sido propostos na literatura, envolvendo diferentes tecnologias, tais como a utilização de sistemas de adsorção com alumina ativada (GHORAI e PANT, 2005; KAMBLE et al., 2010; CHAUHAN et al., 2007), carvão ativado de osso (TCHOMGUI-KAMGA et al., 2010; COSTA et al., 2008; ABE et al., 2004), resinas de troca iônica (CASTEL et al., 2000), além de sistemas de osmose reversa (SEHN, 2008) e diálise (AMOR et al., 2001). Mais recentemente, Kumar et al. (2010), e Ansari et al. (2011), aplicaram, também, a nanotecnologia para a confecção de nano partículas de alumínio e carvão, respectivamente, em sistemas de desfluoretação.

Uma revisão detalhada sobre diferentes procedimentos de desfluoretação de águas naturais foi apresentada por Meenakshi e Maheshwari (2006), e posteriormente Bhatnagar et al. (2011), onde os autores destacam que a seleção do melhor procedimento a ser adotado depende de fatores químicos, geográficos e econômicos. Ou seja, um procedimento adequado para uma determinada comunidade pode ser pouco eficiente em outras localidades. Entretanto, nenhum dos procedimentos de desfluoretação citados na literatura resultou até hoje na confecção de um sistema de uso doméstico com baixo custo, fácil operação e manutenção (BHATNAGAR et al., 2011).

Neste contexto, o objetivo principal deste estudo foi o desenvolvimento de um protótipo de filtro, utilizando carvão ativado de osso, para a desfluoretação parcial de águas subterrâneas até níveis adequados ao consumo humano, tendo como condições de compromisso a eficiência, a segurança, e o baixo custo de construção e manutenção.

\section{MATERIAIS E METODOS}

O sistema para desfluoretação parcial de águas subterrâneas, proposto neste estudo, foi construído utilizando tubulações de PVC (policloroeteno) com diâmetro interno de $97 \mathrm{~mm}$ e carvão ativado de osso como meio adsorvente de íons fluoreto (Figura 1), conforme descrito em Costa et al. (2008).

Ao todo, foram construídos sete protótipos de filtros (identificados como F1, F2, F3, F4, F5, F6 e F7), utilizando diferentes volumes de carvão ativado de osso como meio adsorvente. A Tabela 1 apresenta as principais características construtivas dos sete protótipos, os quais foram submetidos a um extenso programa de monitoramento. Os protótipos F1, F2 e F3 são réplicas, construídas para avaliação da reprodutibilidade dos resultados.

Os protótipos operaram em fluxo ascendente e a pressão da rede hidráulica onde foram instalados foi de $70 \pm 2 \mathrm{kPa}$ (10 $\pm 0,3 \mathrm{PSI})$. Resultados de perda de carga indicaram valores da ordem de 20 a $35 \%$.

O carvão ativado de osso com diâmetro de partícula entre 8 e 30 mesh foi fornecido pela Bonechar Carvão Ativado do Brasil Ltda, Maringá, PR, Brasil (www.bonechar.com.br). Nenhum tratamento adicional foi aplicado ao carvão, sendo assim, utilizado nas condições em que foi fornecido pela indústria. 


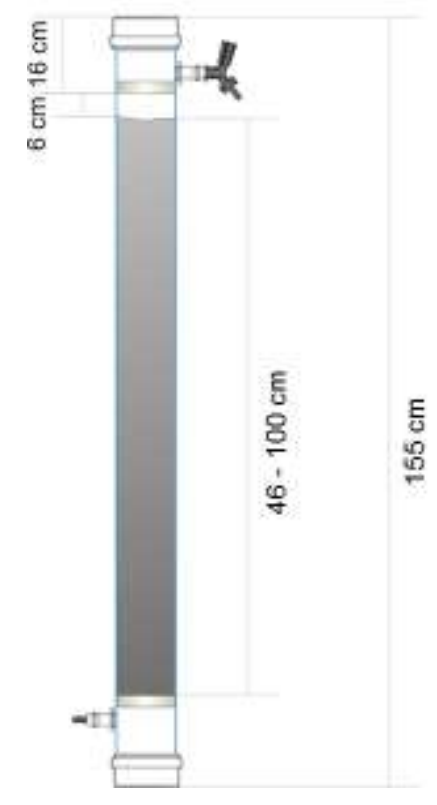

Figura 1 - Diagrama do protótipo de filtro Figure 1 - Diagram of the prototype filter

Tabela 1 - Características construtivas dos protótipos de filtros

Table 1 - Construction characteristics of the prototypes filters

\begin{tabular}{lccccccc}
\hline \multicolumn{1}{c}{ Parâmetros } & F1 & F2 & F3 & F4 & F5 & F6 & F7 \\
\hline Altura total do protótipo, cm & 150 & 150 & 150 & 150 & 150 & 150 & 150 \\
Altura da coluna do meio adsorvente, cm & 46 & 46 & 46 & 60 & 76 & 90 & 100 \\
Diâmetro interno do tubo de PVC, $\mathrm{mm}$ & 94 & 94 & 94 & 94 & 94 & 94 & 94 \\
Diâmetro externo do tubo de PVC, $\mathrm{mm}$ & 100 & 100 & 100 & 100 & 100 & 100 & 100 \\
Volume de meio adsorvente, $\mathrm{L}\left(\mathrm{d}=0,66 \mathrm{~kg} \mathrm{~L}^{-1}\right)$ & 2,91 & 3,08 & 2,98 & 4,40 & 5,40 & 6,30 & 7,70 \\
Massa de meio adsorvente, $\mathrm{kg}$ & 1,92 & 2,03 & 1,97 & 2,90 & 3,56 & 4,16 & 5,08 \\
\hline
\end{tabular}

Os filtros auxiliares de polipropileno de $25 \mathrm{~mm}$ de espessura, $93 \mathrm{~mm}$ de diâmetro e $25 \mu \mathrm{m}$ de porosidade foram fornecidos pela Pentair Hidro Filtros do Brasil Indústria e Comércio de Filtros Ltda., Caxias do Sul, RS, Brasil (http://www.pentairhidrofiltros.com.br/). Esses filtros têm a função de conter adequadamente o carvão ativado de osso dentro dos protótipos de filtração, permitindo uma melhor difusão da água no meio adsorvente e reduzindo a turbidez da água tratada.

Os protótipos foram instalados em um sistema alternativo de abastecimento, no Município de Santa Cruz do Sul, RS, cuja concentração de íons fluoreto varia entre 3,5 e $4,0 \mathrm{mg} \mathrm{L}^{-1}$. Além da cloração, nenhum outro tratamento prévio foi aplicado à água antes da filtração pelos protótipos.

Os sistemas foram operados com uma vazão de $180 \pm 20 \mathrm{~L} \mathrm{~h}^{-1}$, suficiente para atender as necessidades de uso doméstico. Diariamente, foram tratados 10 litros de água, o suficiente para abastecimento diário de uma família de até cinco pessoas, segundo dados da Organização Mundial da Saúde (WHO, 2008).
Após a instalação dos protótipos foi elaborado um cronograma de monitoramento da qualidade da água tratada, bem como da qualidade da água subterrânea bruta. Após a instalação, para os filtros F1, F2 e F3, os primeiros 10 litros de água tratada foram descartados para eliminação de extraíveis originários do carvão. Para os demais protótipos este volume foi de 50 litros.

Durante o desenvolvimento deste estudo a concentração de íons fluoreto foi monitorada, continuamente, na água subterrânea bruta e na tratada pelos sete filtros de desfluoretação. Periodicamente, foram coletadas amostras (quatro ao todo) para avaliação da qualidade da água (águas subterrânea bruta e tratada), em que foram determinados os seguintes parâmetros: alcalinidade bicarbonatos, alcalinidade carbonatos, alcalinidade hidróxidos, cádmio, chumbo, cálcio, cloretos, cloro residual livre, condutividade elétrica, cor aparente, cromo total, dureza total, ferro total, fluoretos, fósforo total, magnésio, manganês, mercúrio, níquel, nitrato, nitrito, potássio, sólidos totais dissolvidos, sódio, sulfato, turbidez, zinco e coliformes termotolerantes. 
As quatro amostras coletadas em cada sistema de filtração foram identificadas como A1 (coletadas imediatamente após a instalação do filtro) e, A2, A3 e A4, distribuídas em diferentes momentos da vida útil destes protótipos.

As amostras da água subterrânea bruta foram coletadas diretamente no reservatório principal, após cloração com hipoclorito de sódio.

As metodologias analíticas utilizadas na coleta das amostras e nas determinações físicas e químicas e microbiológicas encontram-se descritas em APHA (2005).

As propriedades organolépticas da água tratada foram avaliadas através de quatro diferentes testes sensoriais: teste de ordenação (ABNT, 1994), teste de preferência (ABNT, 1994), teste de odor (CORSAN, 2003a) e teste de sabor (CORSAN, 2003b).

Os testes sensoriais foram realizados com um total de 308 julgadores, todos convidados a participar de forma livre e esclarecida. Este estudo foi conduzido de forma a comparar cinco diferentes amostras de água: água da rede pública (ARP), água subterrânea bruta (ASB), águas subterrâneas tratadas pelos filtros de carvão ativado de osso (AST1 e AST2) e água engarrafada (AE), conforme demonstrado na Tabela 2. As amostras foram armazenadas a temperatura ambiente (aproximadamente $20^{\circ} \mathrm{C}$ ), em recipientes plásticos livres de qualquer atributo que pudesse interferir no odor ou sabor das mesmas. Na Tabela 3 estão sintetizadas as características metodológicas de cada um dos testes sensoriais.

$\mathrm{Na}$ interpretação dos resultados obtidos nos testes de ordenação e de preferência foi utilizado o teste de Friedman, GraphPad Instat, versão 3.0, 32 bit for Win 95/NT, utilizando a Tabela de Newel e MacFarlane, conforme NBR 13170, com nível de confiança de $95 \%$. No teste de aceitabilidade a interpretação dos resultados foi realizada através do teste de Turkey, GraphPad Instat, versão 3.0, 32 bit for Win 95/NT.

Para o teste de comparação múltipla foram utilizados testes de variância (ANOVA), através do software Microsoft Excel ${ }^{\circledR}$, e no pro- cessamento das informações de odor e de sabor da água foi utilizada a estatística descritiva para a tabulação dos dados e visualização das medidas de tendência central e dispersão.

Para avaliar a eficiência do meio adsorvente, foi monitorada, diariamente, a concentração de íons fluoreto na água filtrada pelos sete protótipos e, na água subterrânea bruta. A partir destes dados, foram determinadas a capacidade de adsorção absoluta (CA) e a capacidade de adsorção relativa (CAR) de íons fluoreto pelo carvão ativado de osso utilizando as equações 1 e 2 .

$$
\begin{aligned}
& C A=\left(C_{0}-C_{i}\right) \times V_{A T} \\
& C A R=\frac{\left(C_{0}-C_{i}\right) \times V_{A T}}{V_{M A} \text { ou } M_{M A}}
\end{aligned}
$$

Onde:

CA - capacidade de adsorção absoluta (mg)

CAR - capacidade de adsorção relativa $\left(\mathrm{mg} \mathrm{L}^{-}\right.$ ${ }^{1}$ ou $\mathrm{mg} \mathrm{kg}^{-1}$ )

$\mathrm{C}_{0}$ - concentração de íons fluoreto na água bruta (mg L $\left.\mathrm{L}^{-1}\right)$

$\mathrm{C}_{\mathrm{i}}$ - concentração de íons fluoreto na água tratada $\left(\mathrm{mg} \mathrm{L}^{-1}\right)$

$\mathrm{V}_{\mathrm{AT}}$ - volume de água tratada $(\mathrm{L})$

$\mathrm{V}_{\mathrm{MA}}$ - volume de meio adsorvente (L)

$\mathrm{M}_{\mathrm{MA}}$ - massa de meio adsorvente $(\mathrm{kg})$

\section{RESULTADOS E DISCUSSÕES}

Os resultados obtidos na caracterização da água subterrânea utilizada neste estudo indicaram se tratar de uma água alcalina, rica em íons bicarbonatos e sódio, caracterizando-se, como uma água bicarbonatada sódica (Tabela 4). Entre todos os parâmetros avaliados, apenas a concentração de íons fluoreto $\left(3,6 \pm 0,3 \mathrm{mg} \mathrm{L}^{-1}, \mathrm{n}=4\right)$ apresentou-se em desacordo com os padrões estabelecidos para consumo humano segundo a Portaria $\mathrm{n}^{\circ}$ 2914 do Ministério da Saúde (BRASIL, 2011). Resultados semelhantes $\left(3,7 \pm 0,2 \mathrm{mg} \mathrm{L}^{-1}, \mathrm{n}=3\right)$ foram obtidos por Costa et al. (2008).

Tabela 2 - Origem das amostras para os testes sensoriais

Table 2 - Origin of samples for the sensory tests

\begin{tabular}{ll}
\hline \multicolumn{1}{c}{ Amostra } & \multicolumn{1}{c}{ Origem da amostra } \\
\hline Água da rede pública (ARP) & Companhia Riograndense de Saneamento - CORSAN \\
Água subterrânea bruta (ASB) & Poço tubular profundo \\
Água subterrânea tratada 1 (AST1) & Filtro de carvão ativado de osso em fase inicial de operação \\
Água subterrânea tratada 2 (AST2) & Filtro de carvão ativado de osso em fase final de operação \\
Água engarrafada (AE) & Adquirida em comércio local \\
\hline
\end{tabular}


Tabela 3 - Características metodológicas dos testes sensoriais

Table 3 - Methodological characteristics of sensory tests

\begin{tabular}{lll}
\hline \multicolumn{1}{c}{ Teste sensorial } & \multicolumn{1}{c}{ Metodologia } & \multicolumn{1}{c}{ Total de julgadores } \\
\hline Ordenação & ABNT (1994), NBR 13170 & 108 (47 homens e 61 mulheres) \\
Preferência & ABNT (1994), NBR 13170 & 100 (55 homens e 45 mulheres) \\
Determinação de odor & CORSAN (2003a), FQ-S 004A & 50 (26 homens e 24 mulheres) \\
Determinação de sabor & CORSAN (2003b), FQ-S 016 & 50 (sem identificação) \\
\hline
\end{tabular}

Tabela 4 - Resultados obtidos no monitoramento da água subterrânea bruta (ASB)

Table 4 - Results obtained in the monitoring of raw groundwater (ASB)

\begin{tabular}{|c|c|c|c|c|c|}
\hline \multirow[t]{2}{*}{ Parâmetro } & \multirow[t]{2}{*}{ VMP } & \multicolumn{4}{|c|}{ Amostras } \\
\hline & & A1 & $\mathbf{A} 2$ & A3 & A4 \\
\hline $\begin{array}{l}\text { Alcalinidade bicarbonatos, } \mathrm{mg} \mathrm{L}^{-1}(\mathrm{Ca}- \\
\left.\mathrm{CO}_{3}\right)\end{array}$ & $*$ & 147,3 & 147,3 & 153,9 & 134,4 \\
\hline Alcalinidade carbonatos, $\mathrm{mg} \mathrm{L}^{-1}\left(\mathrm{CaCO}_{3}\right)$ & $*$ & 37,4 & 37,4 & 28,6 & 29,8 \\
\hline Alcalinidade hidróxidos, $\mathrm{mg} \mathrm{L}^{-1}\left(\mathrm{CaCO}_{3}\right)$ & $*$ & $<0,1$ & $<0,1$ & $<0,1$ & $<0,1$ \\
\hline Cádmio, $\mu \mathrm{g} \mathrm{L}^{-1}$ & 5 & $<0,1$ & $<0,1$ & $<0,1$ & $<0,1$ \\
\hline Chumbo, $\mu \mathrm{g} \mathrm{L}^{-1}$ & 10 & $<1,0$ & $<1,0$ & $<1,0$ & $<1,0$ \\
\hline Cálcio, $\mathrm{mg} \mathrm{L}^{-1}$ & $*$ & 0,87 & 0,72 & 0,40 & 0,8 \\
\hline Cloreto, $\mathrm{mg} \mathrm{L}^{-1}\left(\mathrm{Cl}^{-}\right)$ & 250 & 10,4 & 10,9 & 15,6 & 12,0 \\
\hline Cloro residual livre, $\mathrm{mg} \mathrm{L}^{-1}\left(\mathrm{Cl}_{2}\right)$ & 5,0 & 0,38 & 0,09 & 0,16 & 0,43 \\
\hline Condutividade elétrica, $\mu \mathrm{S} \mathrm{cm}^{-1}$ & $*$ & 473 & 474 & 497 & 480 \\
\hline Cor aparente, $\mathrm{uH}$ & 15 & $<2$ & $<2$ & $<2$ & $<2$ \\
\hline Cromo total, $\mathrm{mg} \mathrm{L}^{-1}$ & 0,05 & $<0,03$ & $<0,03$ & $<0,03$ & $<0,03$ \\
\hline Dureza total, $\mathrm{mg} \mathrm{L}^{-1}\left(\mathrm{CaCO}_{3}\right)$ & 500 & 2,3 & 1,9 & 1,1 & 2,0 \\
\hline Ferro total, $\mathrm{mg} \mathrm{L}^{-1}$ & 0,3 & $<0,02$ & $<0,02$ & $<0,02$ & $<0,02$ \\
\hline Fluoreto, $\mathrm{mg} \mathrm{L}^{-1}$ & 1,5 & 3,7 & 3,5 & 3,8 & 3,2 \\
\hline Fósforo total, mg L ${ }^{-1}(\mathrm{P})$ & $*$ & 0,02 & 0,07 & 0,14 & 0,8 \\
\hline Magnésio, $\mathrm{mg} \mathrm{L}^{-1}$ & $*$ & 0,03 & 0,04 & 0,03 & 0,03 \\
\hline Manganês, mg L ${ }^{-1}$ & 0,1 & $<0,02$ & $<0,02$ & $<0,02$ & $<0,02$ \\
\hline Mercúrio, $\mu \mathrm{g} \mathrm{L}^{-1}$ & 1 & $<0,05$ & $<0,05$ & $<0,05$ & $<0,05$ \\
\hline Níquel, $\mathrm{mg} \mathrm{L}^{-1}$ & $0,02 *, \delta$ & $<0,02$ & $<0,02$ & $<0,02$ & $<0,02$ \\
\hline Nitrato, $\mathrm{mg} \mathrm{L}^{-1}\left(\mathrm{~N}-\mathrm{NO}_{3}^{-}\right)$ & 10 & 0,2 & 0,1 & 0,2 & 0,2 \\
\hline Nitrito, $\mathrm{mg} \mathrm{L}^{-1}\left(\mathrm{~N}-\mathrm{NO}_{2}^{-}\right)$ & 1 & $<0,01$ & $<0,01$ & $<0,01$ & $<0,01$ \\
\hline $\mathrm{pH}$ & 6,0 a 9,5 & 9,2 & 9,2 & 9,1 & 9,2 \\
\hline Potássio, $\mathrm{mg} \mathrm{L}^{-1}$ & $*$ & $<0,02$ & $<0,02$ & $<0,02$ & $<0,02$ \\
\hline Sódio, $\mathrm{mg} \mathrm{L}^{-1}$ & 200 & 154,4 & 154,4 & 154,4 & 136,2 \\
\hline Sólidos totais dissolvidos, $\mathrm{mg} \mathrm{L}^{-1}$ & 1.000 & 295,5 & 299,0 & 295,0 & 250,5 \\
\hline Sulfato, $\mathrm{mg} \mathrm{L}^{-1}\left(\mathrm{SO}_{4}{ }^{2-}\right)$ & 250 & 16,3 & 15,9 & 39,0 & 34,3 \\
\hline Turbidez, uT & 5 & $<0,02$ & $<0,02$ & $<0,02$ & $<0,02$ \\
\hline Zinco, $\mathrm{mg} \mathrm{L}^{-1}$ & 5 & $<0,02$ & $<0,02$ & $<0,02$ & $<0,02$ \\
\hline $\begin{array}{l}\text { Coliformes termotolerantes, NMP } 100 \mathrm{~mL} \\
\text { }\end{array}$ & Ausente & Ausente & Ausente & Ausente & Ausente \\
\hline
\end{tabular}

Onde: (VMP) Valor máximo permitido pela Portaria $\mathrm{n}^{\circ}$ 2914, do Ministério da Saúde; $\left(^{*}\right)$ parâmetros não citados pela Portaria $\mathrm{n}^{\circ}$ 2914, do Ministério da Saúde.

Os resultados obtidos no monitoramento da qualidade da água tratada permitiram identificar um aumento na concentração de sólidos totais dissolvidos, correlacionada aos íons de fósforo, sódio e potássio extraídos do meio adsorvente.
Além disto, o caráter alcalino do carvão ativado de osso resultou no aumento da alcalinidade por carbonatos e, concomitantemente, do $\mathrm{pH}$ da água tratada (Figura 2). 

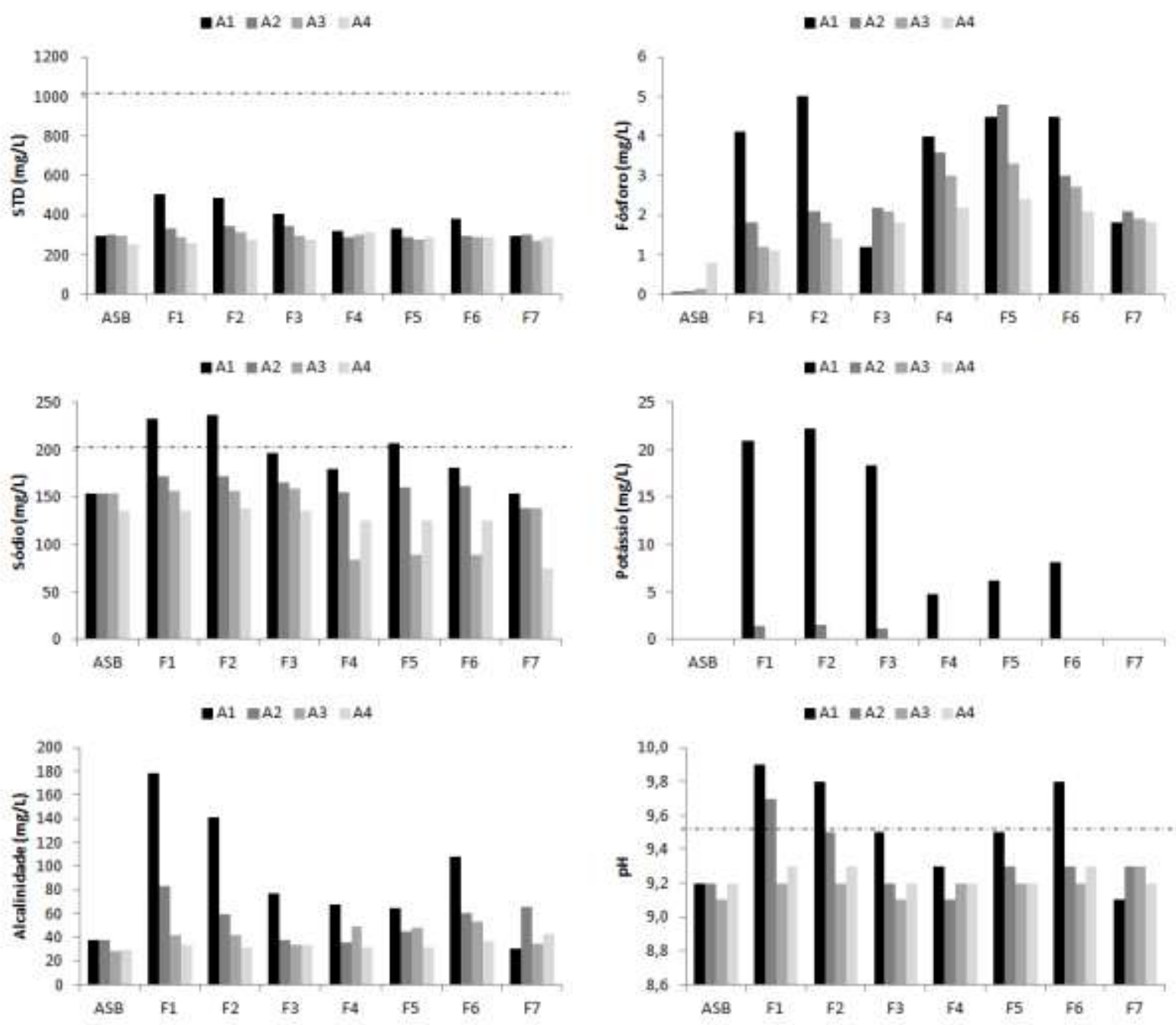

Figura 2 - Concentração de sólidos totais dissolvidos (STD), fósforo total, sódio, potássio, alcalinidade por carbonatos e $\mathrm{pH}$ nas quatro amostras coletadas da água tratada pelos protótipos F1 a F7 e da água subterrânea bruta (ASB). A linha pontilhada representa o valor máximo permitido (VMP) pela Portaria n ${ }^{\circ}$ 2914, do Ministério da Saúde, Brasil

Figure 2 - Concentration of total dissolved solids (TDS), total phosphorus, sodium, potassium, carbonate alkalinity and $\mathrm{pH}$ for the four treated samples water for the prototypes F1 to F7, and the sample of raw groundwater (ASB). The dotted line represents the maximum allowable value (PMV) by Ordinance $\mathrm{n}^{\circ} 2914$, the Ministry of Health, Brazil

Este fenômeno foi mais evidente nos protótipos F1, F2 e F3, e nas alíquotas coletadas logo após a sua instalação (amostra A1). Nos filtros F4, F5, F6 e F7, nos quais um volume superior de água para purga foi utilizado (50 litros), não foram identificados aumentos significativos $(\mathrm{p}>0,05)$ na concentração de extraíveis. Entre todos os parâmetros físicos e químicos determinados, os resultados para sódio e $\mathrm{pH}$ foram os mais preocupantes, uma vez que extrapolaram os limites estabelecidos para abastecimento público na Portaria $n^{\circ}$ 2914, do Ministério da Saúde (BRASIL, 2011).

Cabe salientar, também, que a água subterrânea utilizada neste estudo apresentou, naturalmente, valores elevados para $\mathrm{pH}(9,2 \pm 0,1, \mathrm{n}=$
4) e sódio $\left(149,9 \pm 9,1 \mathrm{mg} \mathrm{L}^{-1}, \mathrm{n}=4\right)$.

Os resultados obtidos identificaram, também, a remoção completa do cloro livre residual da água, cujos resultados foram próximos, ou mesmo inferiores, ao limite de detecção do método analítico $\left(0,05 \mathrm{mg} \mathrm{L}^{-1}\right)$.

Desta forma, surge a necessidade de que essa água tratada seja consumida logo após sua filtração, como ocorre nos filtros de uso doméstico, evitando-se contaminações posteriores devido ao seu armazenamento, principalmente se os reservatórios forem externos.

Quanto às características microbiológicas da água tratada, foram encontrados resultados positivos para contaminação fecal em apenas 3 das 24 amostras coletadas. Estes resultados foram 
obtidos nos filtros F2 (amostra A4), F3 (amostra A3) e F4 (amostra A3). Devido à pontualidade destes resultados, acredita-se na possibilidade de contaminação externa, associada à amostragem, visto que os filtros permaneceram expostos ao ambiente durante os testes experimentais.

Os resultados obtidos nos testes sensoriais indicaram uma boa receptividade para o consumo da água tratada pelos protótipos de filtros de carvão ativado de osso. Na maioria dos experimentos, as amostras AST1 e AST2 figuraram entre as de melhor nota, havendo uma relação direta com a aceitação da água por parte dos consumidores.

Os testes de ordenação revelaram diferenças significativas entre as amostras pesquisadas $(\mathrm{p}<0,05)$, indicando que a amostra de água engarrafada (AE) é significativamente diferente (mais objetável), do que as amostras de água tratada (AST1 e AST2). Os resultados dos testes sensoriais de preferência indicaram que a amostra de água da rede pública (ARP) é significativamente menos preferida $(p<0,05)$ em relação à amostra de água tratada pelo filtro AST2.

No que diz respeito aos resultados de odor, observou-se que a maioria $(94,4 \pm 3,8 \%)$ dos julgadores não identificou nenhum odor nas amostras pesquisadas, destacando-se a amostra AST1 onde nenhum dos 50 julgadores atribuiu odor algum. Quanto aos resultados dos testes organolépticos de sabor, apesar das amostras AST1 e AST2 apresentarem-se como as mais objetáveis, não foram identificadas diferenças significativas $(p>0,05)$ em relação às demais amostras.

Quanto a vida útil dos protótipos, esta foi definida em função do instante em que os resultados na água tratada atingiram valores de concentração de íons fluoreto superiores a $1,5 \mathrm{mg} \mathrm{L}^{-1}$, limite máximo estabelecido pela Portaria $\mathrm{n}^{\circ} 2914$, do Ministério da Saúde, indicando a saturação do meio adsorvente para os objetivos deste estudo. Neste instante, a razão $\mathrm{C}_{\mathrm{i}} / \mathrm{C}_{0}$ corresponde a aproximadamente 0,41 , onde $\mathrm{C}_{0}$ é a concentração inicial de íons fluoreto $\left(3,6 \pm 0,3 \mathrm{mgL}^{-1}, \mathrm{n}=4\right)$, e $\mathrm{C}_{\mathrm{i}}$ a concentração de íons fluoreto no tempo $i$, que corresponde $1,5 \mathrm{mgL}^{-1}$. Os resultados do monitoramento da concentração de íons fluoreto da água tratada pelos sete protótipos de filtros estão apresentados na Figura 3.

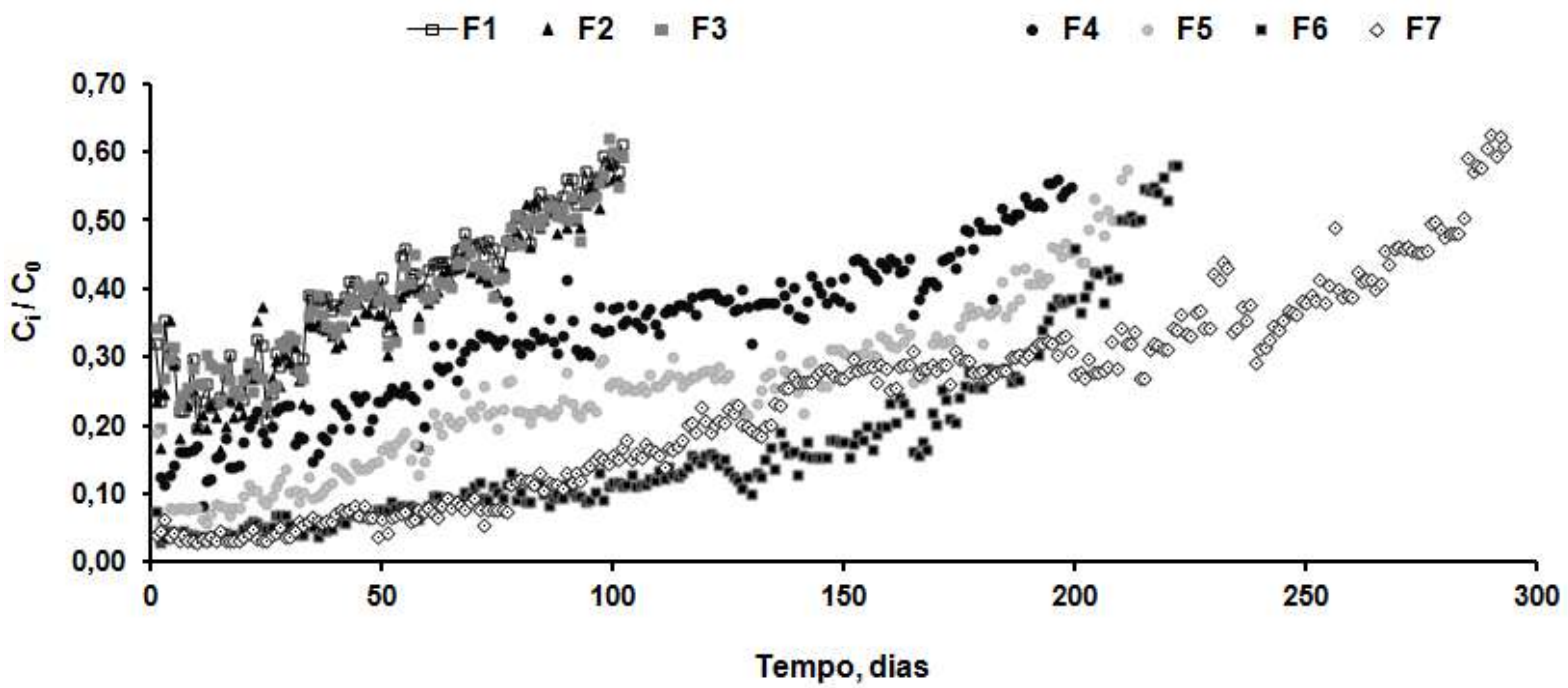

Figura 3 - Resultados do monitoramento da concentração de íons fluoreto na água tratada pelos sete protótipos de filtro

Figure 3 - Results of monitoring the concentration of fluoride ions in the water treated by the seven prototypes filter 


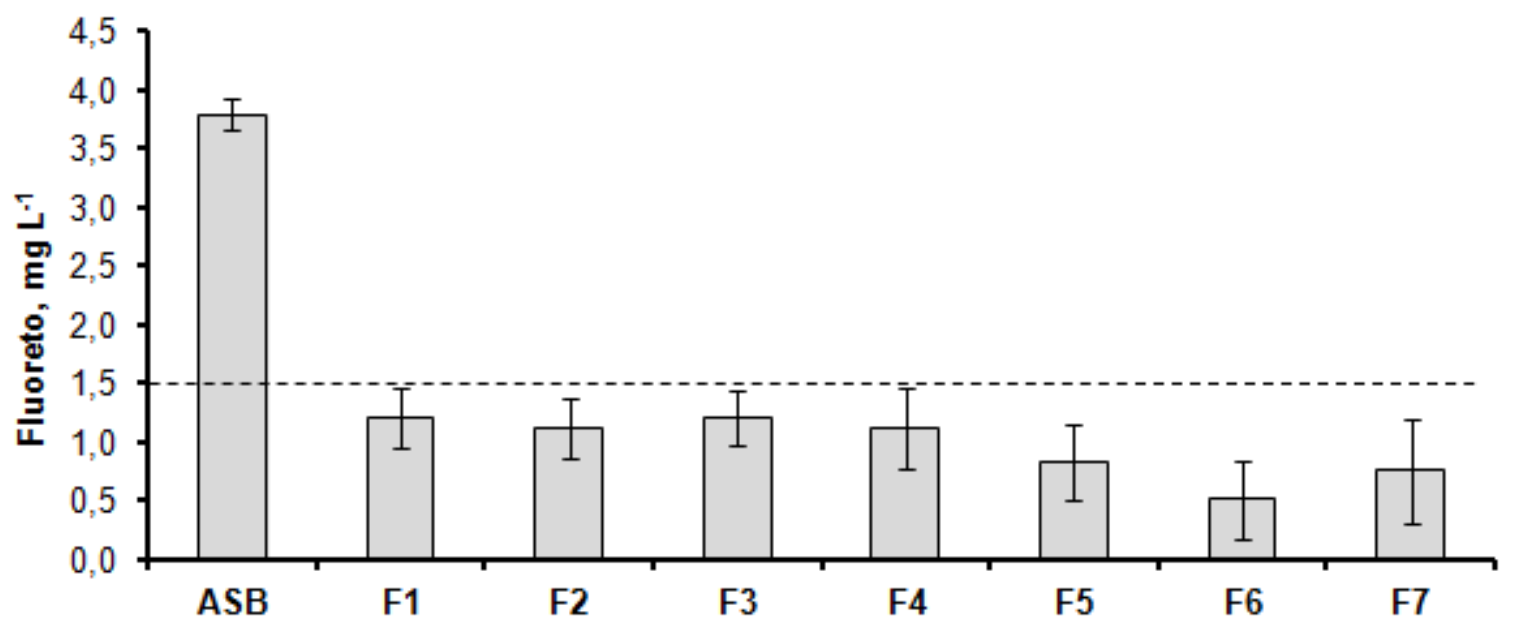

Figura 4 - Valores médios ( \pm desvio-padrão) para íons fluoreto obtidos no monitoramento da água bruta $(n=48)$ e na água tratada pelos protótipos F1 $(n=53), F 2(n=65), F 3(n=62), F 4(n=150), F 5$ $(n=194)$, F6 $(n=204)$ e F7 ( $n=250)$. A linha pontilhada representa o valor máximo permitido (VMP) pela Portaria $n^{\circ} 2914$, do Ministério da Saúde, Brasil

Figure 4 - Mean values ( \pm standard deviation) for fluoride ions obtained in the monitoring of raw water $(n=$ $48)$ and the water treated by the prototypes F1 $(n=53), F 2(n=65), F 3(n=62), F 4(n=150), F 5$ $(\mathrm{n}=194), \mathrm{F} 6(\mathrm{n}=204)$ and F7 $(\mathrm{n}=250)$. The dotted line represents the maximum allowable value (PMV) by Ordinance $n^{\circ} 2914$, the Ministry of Health, Brazil

$\mathrm{O}$ ponto de ruptura (breakpoint) foi estabelecido no instante em que $\mathrm{C}_{\mathrm{i}} / \mathrm{C}_{0}=0,1$, ou seja, no instante em que a concentração de íons fluoreto na água tratada atinge $10 \%$ da concentração inicial $\left(\mathrm{C}_{0}\right)$.

Os protótipos F1, F2 e F3, com volume de carvão da ordem de três litros, não apresentaram variação significativa $(\mathrm{p}<0,05)$ na eficiência de adsorção, indicando uma vida útil de dois meses de operação (média de $60 \pm 6$ dias). Estes resultados atestam que os procedimentos construtivos e de operação, bem como de eficiência do sistema, foram uniformes, permitindo uma boa reproduti- bilidade dos resultados. Os protótipos F4, F5, F6 e F7, confeccionados com volumes diferentes de carvão ativado de osso, apresentaram resultados de vida útil da ordem de 150, 194, 204 e 250 dias, respectivamente.

Os pontos de ruptura dos protótipos F1, $\mathrm{F} 2$, F3 e F4, não puderam ser determinados, pois a concentração de íons fluoreto na água tratada $\left(\mathrm{C}_{\mathrm{i}}\right)$ foi, sempre, superior a $10 \%$ da concentração inicial $\left(\mathrm{C}_{0}\right)$, atestando que estes protótipos foram subdimensionados. A Tabela 5 resume os resultados de ponto de ruptura e de saturação dos sete protótipos.

Tabela 5 - Resultados operacionais apresentados pelos sete protótipos de filtros

Table 5 - Operating results for the seven prototypes filters

\begin{tabular}{lccccccc}
\hline & F1 & F2 & F3 & F4 & F5 & F6 & F7 \\
\hline Tempo de saturação, dias & 53 & 65 & 62 & 150 & 194 & 204 & 250 \\
Ponto de ruptura, dias & ND & ND & ND & ND & 27 & 71 & 78 \\
Volume de água tratada (V & 530 & 650 & 620 & 1.500 & 1.940 & 2.040 & 2.500 \\
Capacidade de adsorção (CA), mg & 1.524 & 1.657 & 1.677 & 4.479 & 5.621 & 6.621 & 7.883 \\
Capacidade de adsorção relativa (CAR), $\mathrm{mg} \mathrm{L}^{-1}$ & 524 & 538 & 563 & 1.018 & 1.041 & 1.051 & 1.024 \\
Capacidade de adsorção relativa (CAR), $\mathrm{mg} \mathrm{kg}^{-1}$ & 794 & 815 & 853 & 1542 & 1577 & 1592 & 1552 \\
\hline \multicolumn{1}{l}{ Onde: ND - não determinado. } & & & & & & &
\end{tabular}

Na Figura 4 é apresentado um comparativo entre a concentração média de íons fluoreto na água subterrânea bruta e na água tratada, nos sete protótipos avaliados, durante a vida útil destes sistemas.

Os resultados da CAR dos filtros F1, F2 e F3 foram significativamente semelhantes $(\mathrm{p}<0,05)$, indicando, como média, que 1 litro de carvão ativado de osso adsorve $541 \pm 20 \mathrm{mg} \mathrm{L}^{-1} \mathrm{de}$ íons fluoreto, nas condições de operação deste estudo.

Já, os protótipos F4, F5, F6 e F7, apresentaram resultados de CAR significativamente superiores aos demais (média $=1.033 \pm 15 \mathrm{mg} \mathrm{L}^{-1}$ ), e 
não apresentaram diferenças significativas $(p<0,05)$ na CAR entre si. Resultados semelhantes foram obtidos em outros estudos, como no de Abe et al. (2004), que descrevem este material como o de maior capacidade de adsorção, quando comparado a outros materiais carbonosos.

Estes resultados indicaram uma correlação linear (coeficiente de correlação de Pearson), extremamente significante, entre a capacidade de adsorção absoluta (CA) e o volume de carvão utilizado na confecção dos protótipos F4 a F7 ( $\mathrm{r}=$ $0,9981, \mathrm{p}=0,0019)$.

Neste contexto, observou-se que acima de $60 \mathrm{~cm}$ de altura de coluna do meio adsorvente (protótipos F4 - F7), e considerando como constantes a concentração de íons fluoreto na água bruta, o tamanho de partícula do meio adsorvente, a vazão de água afluente e o diâmetro da coluna; o volume (ou massa) de meio adsorvente ficou diretamente correlacionado ao volume de água tratada. Os resultados de correlação (coeficiente de correlação de Pearson) indicaram uma correlação significativa entre estas duas propriedades ( $\mathrm{r}=$ 0,9839, $\mathrm{p}=0,0002$ ).

A partir dos resultados dos protótipos $\mathrm{F} 4$, F5, F6, F7 foi produzido um modelo de regressão linear correlacionando o volume de meio adsorvente utilizado $\left(\mathrm{V}_{\mathrm{MA}}\right)$ e o volume de água tratada $\left(\mathrm{V}_{\mathrm{AT}}\right)$ (até a saturação do sistema), Figura 5.

$\mathrm{O}$ modelo de regressão obtido $\left(\mathrm{V}_{\mathrm{AT}}=\right.$ $\left.288,1 \mathrm{~V}_{\mathrm{MA}}+280,7\right)$ determinou que, nas condições de operação aplicadas aos protótipos, para a produção de $1800 \mathrm{~L}$ de água tratada são necessários 5,3 L de carvão ativado de osso ou $3,5 \mathrm{~kg}$ se considerada a equação em função da massa de meio adsorvente $\left(\mathrm{V}_{\mathrm{AT}}=436,5 \mathrm{M}_{\mathrm{MA}}+280,7\right)$. Este volume de água é suficiente para atender as necessidades de consumo de um família de até 5 indivíduos adultos, por um período de até 6 meses (WHO, 2008).

Assim, a Tabela 6 resume as principais características construtivas e de operação de um sistema para adsorção de íons fluoreto, para uso doméstico e utilizando carvão ativado de osso.

Tabela 6 - Características construtivas e de operação de um sistema de adsorção de íons fluoreto utilizando carvão ativado de osso como meio adsorvente

Table 6 - Construction and operation characteristics of a system of fluoride ion adsorption using bone charcoal activated as adsorbent

\begin{tabular}{lc}
\hline \multicolumn{1}{c}{ Características } & Valores \\
\hline Altura da coluna do meio adsorvente, cm & 60 \\
Diâmetro da coluna de meio adsorvente, mm & 94 \\
Volume de carvão ativado de osso, L & 5,3 \\
Massa de carvão ativado de osso, kg & 3,5 \\
Diâmetro de partícula do meio adsorvente, mesh & $8-30$ \\
Concentração de íons fluoreto na água bruta, mg L & $\leq 3,8$ \\
Concentração de íons fluoreto na água tratada, $\mathrm{mg} \mathrm{L}^{-1}$ & $\leq 1,5$ \\
Vazão de operação, L h ${ }^{-1}$ & $180 \pm 20$ \\
Volume diário de água tratada, L & 10 \\
Volume total de água tratada, L & 1800 \\
Vida útil do sistema, dias & 180 \\
\hline
\end{tabular}

O tempo de vida útil máximo foi indicado em função de recomendações usuais para filtros domésticos. Esta manutenção semestral minimiza riscos de entupimento, formação de colônias de microrganismos e, desta forma, reduz riscos de mau funcionamento e contaminação microbiológica do sistema.

Por fim, cabe destacar que neste estudo não foram considerados procedimentos de regeneração do meio adsorvente, uma vez que, devido à pequena quantidade de carvão ativado e seu custo acessível ( $\mathrm{R} \$ 4,00$ por $\mathrm{kg}$ ), assim como para outros filtros de uso doméstico, a regeneração do meio adsorvente não é economicamente favorável.

\section{CONCLUSÕES}

Os resultados obtidos demonstraram a eficiência do carvão ativado de osso na adsorção de íons fluoreto, indicando que sistemas de filtração construídos com $3,5 \mathrm{~kg}$ deste material são adequados para atender as necessidades de consumo de uma família de 5 pessoas, por um período de seis meses, desde que observadas as condições de montagem e operação descritas neste estudo.

Devido às características químicas do carvão ativado de osso, há dissolução de íons sódio, potássio, fósforo e carbonatos na água tratada, principalmente no início do funcionamento 
do sistema. $\mathrm{O}$ excesso destes íons é minimizado a níveis adequados para o consumo humano, pelo simples descarte dos primeiros $50 \mathrm{~L}$ de água.

Finalmente, é importante assinalar que os resultados obtidos neste estudo contribuem para o desenvolvimento de uma tecnologia na área de engenharia de saúde pública, que se caracteriza pelo baixo custo de implantação, operação e manutenção, possibilitando à população melhores condições de acesso a água potável, especialmente em áreas rurais desprovidas de rede pública de abastecimento.

\section{AGRADECIMENTOS}

Os autores agradecem ao apoio da FAPERGS, FUNASA, Secretaria de Ciência Inovação e Desenvolvimento Tecnológico do Estado do Rio Grande do Sul, e ao Fundo de Apoio a Pesquisa da Universidade de Santa Cruz do Sul (FAP-UNISC).

\section{REFERÊNCIAS}

ABE, I.; IWASAKI, S.; TOKIMOTO, T.; KAWASAKI, N.; NAKAMURA, T.; TANADA, S. Adsorption of fluoride ions onto carbonaceous materials, Journal of Colloid and Interface Science, v.275, p.35-39, 2004.

ABNT - Associação Brasileira de Normas Técnicas - NBR 13170: Teste de ordenação em análise sensorial. Rio de Janeiro: ABNT, 7p.,1994.

AMOR, Z.; BARIOU, B.; MAMERI, N.; TAKY, M.; NICOLAS, S.; ELMIDAOUI, A. Fluoride removal from brackish water by electrodialysis. Desalination, v.133, p.215-223, 2001.

ANSARI, M.; KAZEMIPOUR, M.; DEHGHANI, M.; KAZEMIPOUR, M. The defluoridation for drinking water using multi-waled carbon nanotubes. Journal of Fluorine chemistry, v.132, p.516-520, 2011.

APHA - American Public Health Association. Standard methods for the examination of water \& wastewater, Washington: APHA, 21 ed., 1000p., 2005.

BHATNAGAR, A.; KUMAR, E.; SILLANPÄÄ, M. Fluoride removal from water by adsorption $-\mathrm{A}$ review. Chemical Engineering Journal, v.171, p.811-840, 2011.

BRASIL. Ministério da Saúde. Portaria $n^{\circ}$ 2914, de 12 de dezembro de 2011. Dispõe sobre os procedimentos de controle e de vigilância da qualidade da água para consumo humano e seu padrão de potabilidade. Diário Oficial União, Brasília, v. 239, p. 39-46, 14 dez. 2011, Seção 1.

CANGUSSU, M. C. T.; NARVAI, P. C.; FERNANDEZ, R. C.; DJEHIZIAN, V. A. Fluorose dentária no Brasil: uma revisão crítica. Caderno de Saúde Pública, v.18, p.7-15, 2002.

CASTEL, C.; SCHWEIZER M.; SIMONNOT M. O.; SARDIN M. Selective removal of fluoride ions by two-way ionexchange cyclic process. Chemical Engineering Science, v.55, p.3341-3352, 2000.

CASTILHO, L. S.; FERREIRA, E. F.; VELÁSQUEZ, L. N.; FANTINEL, L. M.; PERINI, E. Beliefs and attitudes about endemic dental fluorosis among adolescents in rural Brazil. Revista de Saúde Pública, v.44, p.261-266, 2010.

CATANI, D. B.; HUGO, F. N.; CYPRIANO, S.; SOUSA, M. L.; CURY, J. A. Relationship between fluoride levels in the public water supply and dental fluorosis. Revista de Saúde Pública, v.41, p.732-739, 2007.
CHAUHAN, V. S.; DWIVEDI, P. K.; IYENGAR, L. Investigations on activated alumina based domestic defluoridation units. Journal of Hazardous Materials, B139, p.103-107, 2007.

CORSAN - Companhia Riograndense de Saneamento. Determinação de odor - método de análise sensorial em ETAs, FQS 004A, 2003a.

CORSAN - Companhia Riograndense de Saneamento. Determinação de gosto - método de análise degustativa, FQS 016A, 2003b.

COSTA, A. B.; SOARES, J.; LOBO, E. A.; KIRST, A.; KUNRATH, I. G.; CORD, D. D. Avaliação da qualidade da água tratada por sistemas de filtração utilizando carvão ativado de osso. UNOPAR Científica Ciências Exatas e Tecnológicas, v.7, p.5-12, 2008.

FRAZÃO, P.; PERES, M. A.; CURY, J. A. Drinking water quality and fluoride concentration. Revista de Saúde Pública, v.45, p.964-973, 2011.

GHORAI, S.; PANT, K. K. Equilibrium, kinetics and breakthrough studies for adsorption of fluoride on activated alumina. Separation and Purification Technology, v.42, p.265271, 2005.

KAMBLE, S. P.; DESHPANDE, G.; BARVE, P. P.; RAYALU, S.; LABHSETWAR, N. K.; MALYSHEW, A.; KULKARNI, B. D. Adsorption of fluoride from aqueous solution by alumina of alkoxide nature: Batch and continuous operation. Desalination, v.264, p.15-23, 2010.

KUMAR, E.; BHATNAGAR, A.; KUMAR, U.; SILLAN$\mathrm{PÄ} \ddot{A}$, M. Defluoridation from aqueous solutions by nanoalumina: Characterization and sorption studies. Journal of Hazardous Materials, v.186, p.1042-1049, 2010.

LOBO, E. A.; COSTA, A. B. Análise descritiva da concentração de íons fluoreto em poços artesianos da região do Vale do Rio Pardo e Rio Taquari, RS, Brasil. Revista Tecnológica, v.2, p.29-35, 1998.

MEENAKSHI, R. C.; MAHESHWARI, A. Fluoride in drinking water and its removal. Journal of Hazardous Materials, v.137, p.456-463, 2006.

RIO GRANDE DO SUL. Secretaria Estadual da Saúde. Portaria $\mathrm{n}^{\circ} 10$ de 16 de agosto de 1999. Define teores de concentração do íon fluoreto nas águas para consumo huma- 
no fornecidas por sistemas públicos de abastecimento. Diário Oficial do Estado, Porto Alegre, 27 ago. 1999.

SEHN, P. Fluoride removal with extra low energy reverse osmosis membranes: three years of large scale field experience in Finland. Desalination, v.223, p.73-84, 2008.

TCHOMGUI-KAMGA, E.; NGAMENI, E.; DARCHEN, A. Evaluation of removal efficiency of fluoride from aqueous solution using new charcoals that contain calcium compounds. Journal of Colloid and Interface Science, v. 346, p. 494-499, 2010.

WHO - World Health Organization. Guidelines for drinking-water quality, Recommendations. Geneva: WHO, v.1, 3 ed., 668p., 2008. 